\title{
AUTOMORPHISMS AND TENSOR PRODUCTS OF ALGEBRAS
}

\author{
JOHN W. BUNCE ${ }^{1}$
}

\begin{abstract}
In this note we prove that if $A$ is a complex Banach algebra with identity, then the automorphism on $A \hat{\otimes} A$ determined by $\theta(a \otimes b)=b \otimes a$ is inner if and only if $A=M_{n}(C)$.
\end{abstract}

Let $A$ be a $C^{*}$-algebra and let $A \otimes \otimes^{*} A$ be the $C^{*}$-tensor product of $A$ with itself. Let $\theta$ be the automorphism of $A \otimes^{*} A$ determined by $\theta(a \otimes b)=$ $b \otimes a$ for all $a, b$ in $A$. S. Sakai proved in [3] that $\theta$ is inner if and only if $A$ is the algebra $M_{n}(C)$ of all $n \times n$ complex matrices for some positive integer $n$. In an invited talk at the International Conference on Banach Spaces, Wabash College, June, 1973, Sakai asked if the theorem had an extension to general Banach algebras. In Theorem 1 of this note we prove that if $A$ is a complex Banach algebra with identity of norm one, then the automorphism on $A \hat{\otimes} A$ determined by $\theta(a \otimes b)=b \otimes a$ is inner if and only if $A=M_{n}(C)$. In Theorem 2 we prove that if $A$ is an algebra over an algebraically closed field $F$, then the automorphism $\theta$ as defined above on the algebraic tensor product $A \otimes_{F} A$ is inner if and only if $A=M_{n}(F)$. The proofs of these theorems are much easier than the proof of the $C^{*}$-algebra theorem.

Let $A$ be a Banach algebra with identity $e$; we assume $\|e\|=1$. Let $A \otimes A$ be the algebraic tensor product of the complex vector space $A$ with itself, and let $\dot{A} \hat{\otimes} A$ be the completion of $A \otimes A$ in the greatest crossnorm [4]. The greatest crossnorm on $A \otimes A$ is an algebra norm [1], so $A \hat{\otimes} A$ is a Banach algebra. It is clear that the map $\theta$ from $A \hat{\otimes} A$ to $A \hat{\otimes} A$ determined by $\theta(a \otimes b)=b \otimes a$, for all $a, b$ in $A$, is an automorphism. If $A=M_{n}(C)$, then $A \hat{\otimes} A$ is algebraically isomorphic to $M_{n^{2}}(C)$, and it is well known that every automorphism of $M_{n^{2}}(C)$ is inner.

THEOREM 1. If the automorphism $\theta$ of $A \hat{\otimes} A$ is inner, then $A=M_{n}(C)$ for some positive integer $n$.

Received by the editors August 9, 1973.

AMS (MOS) subject classifications (1970). Primary 46H20, 46M05; Secondary 16A72, 16A40.

Key words and phrases. Banach algebra, tensor product, automorphism.

${ }^{1}$ The author was partially supported by NSF Grant GP-37526. 
Proof. We first prove that $A$ is a simple algebra. Let $I$ be a closed ideal in $A$ with $I \neq A$. Let $p$ be the quotient map from $A$ to $A / I$ and consider the canonical map [5, p. 439] $p \hat{\otimes}$ id: $A \hat{\otimes} A \rightarrow(A \mid I) \hat{\otimes} A$, where id is the identity map of $A$. Then $p \hat{\otimes}$ id is clearly a homomorphism, and by [5, p. 445] $\operatorname{Ker}(p \hat{\otimes} \mathrm{id})=\operatorname{cl}(I \otimes A)$, the closure of the space spanned by the elementary tensors $b \otimes a, b \in I, a \in A$. Since $\theta$ is inner we have that $\theta(\operatorname{cl}(I \otimes A)) \subseteq$ $\operatorname{cl}(I \otimes A)$. If $a \in I$ we then have $\theta(a \otimes e) \in \operatorname{Ker}(p \hat{\otimes} \mathrm{id})$, so ( $p \hat{\otimes} \mathrm{id})(e \otimes a)=$ $(e+I) \otimes a=0$, and $a=0$. Hence $I=\{0\}$ and $A$ has no nontrivial closed ideals, and in fact no nontrivial ideals since $A$ is complete and has an identity. By the classical Wedderburn-Artin theorem [2, Theorem 2.1.8], we now need only show that $A$ is finite dimensional. Let $d \in A \hat{\otimes} A$ implement the inner automorphism $\theta$, so that $b \otimes a=d(a \otimes b) d^{-1}$ for all $a, b \in A$. Now choose $z$ and $w$ in the algebraic tensor product $A \otimes A$ with the property that

$$
\begin{gathered}
\|z-d\|<\left(4\left\|d^{-1}\right\|\right)^{-1}, \quad\|z\|<\|d\|+1, \\
\left\|w-d^{-1}\right\|<(4(\|d\|+1))^{-1} .
\end{gathered}
$$

Let $z=\sum_{i=1}^{r} x_{i} \otimes y_{i}, w=\sum_{j=1}^{s} u_{j} \otimes v_{j}$. Then if $a, b$ are in $A$ we have

$$
\begin{aligned}
\|b \otimes a-z(a \otimes b) w\| \leqq & \left\|b \otimes a-d(a \otimes b) d^{-1}\right\| \\
& +\left\|d(a \otimes b) d^{-1}-z(a \otimes b) d^{-1}\right\| \\
& +\left\|z(a \otimes b) d^{-1}-z(a \otimes b) w\right\|
\end{aligned}
$$

which is less than or equal to $(\|a\|\|b\|) / 2$. Thus for all $a$ in $A$ we have $\|e \otimes a-z(a \otimes e) w\| \leqq\|a\| / 2$, and hence for all $a$ in $A$,

$$
\left\|e \otimes a-\sum x_{i} a u_{j} \otimes y_{i} v_{j}\right\| \leqq\|a\| / 2,
$$

where the sum is over all $1 \leqq i \leqq r, 1 \leqq j \leqq s$.

Now if $f \in A^{*}$, the bilinear function from $A \times A$ to $A$ defined by $(a, b) \rightarrow$ $f(a) b$ determines a linear function $F$ from $A \hat{\otimes} A$ to $A$ with the property that $\|F\|=\|f\|$ and $F(a \otimes b)=f(a) b$ for all $a, b$ in $A$ [5, Proposition 43.12]. Choose $f \in A^{*}$ such that $f(e)=1=\|f\|$ and apply the corresponding $F$ to the equation $(*)$ to obtain

$$
\left\|a-\sum f\left(x_{i} a u_{j}\right) y_{i} v_{j}\right\| \leqq\|a\| / 2,
$$

for all $a \in A$. Now let $H$ be the finite-dimensional space spanned by the set $\left\{y_{i} v_{j}: 1 \leqq i \leqq r, 1 \leqq j \leqq s\right\}$. We have shown that for all $a \in A$, $H \cap B(a,\|a\| / 2) \neq \varnothing$, where $B(a,\|a\| / 2)$ is the closed ball of radius $\|a\| / 2$ and center $a$. But by Riesz's lemma [6, p. 84], this fact forces $H$ to equal $A$. Thus $A$ is finite dimensional. Q.E.D. 
THEOREM 2. If $A$ is an algebra with identity e over an algebraically closed field $F$ and the automorphism of the algebraic tensor product $A \otimes_{F} A$ determined by $\theta(a \otimes b)=b \otimes a$ is inner, then $A=M_{n}(F)$ for some positive integer $n$.

Proof. The proof that $A$ is a simple algebra is almost the same as the proof that $A$ is simple in Theorem 1; we omit the details. Let $d \in A \otimes A$ be such that $b \otimes a=d(a \otimes b) d^{-1}$ for all $a, b \in A$. Let $d=\sum_{i=1}^{n} x_{i} \otimes y_{i}$, where we assume that the set $\left\{x_{i}\right\}$ is linearly independent [4, Lemma 1.1]. We will show that $A$ is the linear span of $\left\{y_{j}: 1 \leqq j \leqq n\right\}$. For $a, b$ in $A$ we have

$$
\left(\sum x_{i} \otimes y_{i}\right) a \otimes b=b \otimes a\left(\sum x_{i} \otimes y_{i}\right) .
$$

Now if for some $a \in A$ and index $i, a y_{i} \notin \operatorname{span}\left\{y_{j}\right\}$, choose $g$ in the algebraic dual $A^{\prime}$ of $A$ such that $g\left(a y_{i}\right)=1, g\left(y_{j}\right)=0$ for all $j$. Let $G: A \otimes A \rightarrow A$ be the linear function determined by the bilinear function $(c, b) \rightarrow g(b) c$ on $A \times A$, set $b=e$ in $\left({ }^{* *}\right)$, and apply $G$ to obtain

$$
\sum g\left(y_{j}\right) x_{j} a=\sum g\left(a y_{j}\right) x_{j} .
$$

Hence $\sum g\left(a y_{j}\right) x_{j}=0$ but $g\left(a y_{i}\right) \neq 0$, which contradicts our assumption that the $\left\{x_{j}\right\}$ were linearly independent. Thus $\operatorname{span}\left\{y_{j}\right\}$ is a left ideal, and a symmetrical argument shows that $\operatorname{span}\left\{y_{j}\right\}$ is a two-sided ideal. But $A$ is simple, so $A=\operatorname{span}\left\{y_{j}\right\}$ and is finite dimensional. The WedderburnArtin theorem again implies that $A=M_{n}(F)$. Q.E.D.

\section{REFERENCES}

1. B. Gelbaum, Tensor products of Banach algebra, Canad. J. Math. 11 (1959), 297-310. MR 21 \#2922.

2. I. N. Herstein, Noncommutative rings, Carus Mathematical Monograph, no. 15, Mathematical Association of America; distributed by Wiley, New York, 1968. MR 37 \#2790.

3. S. Sakai, Automorphisms and tensor products of operator algebras, Amer. J. Math. (to appear).

4. R. Schatten, A theory of cross-spaces, Ann. of Math. Studies, no. 26, Princeton Univ. Press, Princeton, N.J., 1950. MR 12, 186.

5. F. Treves, Topological vector spaces, distributions and kernels, Academic Press, New York, 1967. MR 37 \#726.

6. K. Yosida, Functional analysis, 2nd ed., Die Grundlehren der math. Wissenschaften, Band 123, Academic Press, New York; Springer-Verlag, Berlin and New York, 1968. MR 39 \#741.

Department of Mathematics, University of Kansas, Lawrence, Kansas 66044 